\title{
Increased brain and atrial natriuretic peptides in patients with chronic right ventricular pressure overload: correlation between plasma neurohormones and right ventricular dysfunction
}

\author{
I I Tulevski, M Groenink, E E van der Wall, D J van Veldhuisen, F Boomsma, J Stoker,
} A Hirsch, J S Lemkes, B J M Mulder

\begin{abstract}
Objective-To evaluate the role of plasma neurohormones in the diagnosis of asymptomatic or minimally symptomatic right ventricular dysfunction.

Setting-Tertiary cardiovascular referral centre.

Methods-Plasma brain natriuretic peptide (BNP) and atrial natriuretic peptide (ANP) concentrations were measured in 21 asymptomatic or minimally symptomatic patients with chronic right ventricular pressure overload caused by congenital heart disease, and in seven healthy volunteers. Right ventricular ejection fraction was determined using magnetic resonance imaging.

Results-Right ventricular ejection fraction in the volunteers was higher than in the patients $(69.0(8.2) \% v 58.0(12.0) \%$, respectively; $\mathrm{p}<0.006)$. Left ventricular ejection fraction was $72.3(7.8) \%$ in volunteers and $68.1(11.0) \%$ in patients (NS). There was a significant difference between patients and volunteers in the plasma concentrations of BNP (5.3 (3.5) $v 2.3$ (1.7) pmol/1, respectively; $\mathrm{p}<0.009$ ) and ANP (7.3 (4.5) v 3.6 (1.4) pmol/1; p < 0.05). In both patients and volunteers, mean plasma ANP was higher than mean plasma BNP. Right ventricular ejection fraction was inversely correlated with BNP and ANP (respectively, $r=0.65 ; \mathrm{p}<0.0002$ and $r=0.61 ; \mathrm{p}<0.002)$. There was no correlation between left ventricular ejection fraction and BNP $(r=0.2 ; \mathrm{NS})$ or ANP $(r=0.52$; NS). Similarly, no correlation was shown between the level of right ventricular systolic pressure and either plasma BNP $(r=0.20)$ or plasma ANP $(r=0.07)$. Conclusions-There was a significant inverse correlation between right ventricular ejection fraction and the plasma neurohormones BNP and ANP in asymptomatic or minimally symptomatic patients with right ventricular pressure overload and congenital heart disease. Monitoring changes in BNP and ANP may provide quantitative follow up of right ventricular dysfunction in these patients.
\end{abstract}

(Heart 2001;86:27-30)

Keywords: natriuretic peptides; right ventricle; pressure overload; congenital heart disease

Early diagnosis of right ventricular dysfunction is difficult but very important in patients with chronic right ventricular pressure overload caused by congenital heart defects. ${ }^{1}$ Absence of an accurate measure of right ventricular function makes it impossible to estimate the optimal moment for medical or surgical intervention so as to prevent or delay irreversible right ventricular failure. ${ }^{2}{ }^{3}$ Recent reports have shown that plasma concentrations of atrial natriuretic peptide (ANP) and brain natriuretic peptide (BNP) are raised in patients with asymptomatic left ventricular systolic dysfunction, and these variables are highly accurate for detecting left ventricular failure. ${ }^{4} \mathrm{BNP}$ is a marker of ventricular distension $^{45}$ and is secreted in both atria and ventricles, especially in failing ventricles. ${ }^{6-8}$ In normal subjects ANP is synthesised and secreted almost exclusively in the atrium, but patients with congestive heart failure have increased production in both atria and ventricles in response to increasing atrial stretch. ${ }^{67}$ Previous studies suggest that neurohumoral markers play a role not only in left ventricular dysfunction $^{9}$ but also in right ventricular dysfunction. ${ }^{10}{ }^{11}$ However, their value as markers of right ventricular dysfunction and their relation to other quantitative determinants of right ventricular function in asymptomatic or minimally symptomatic patients with congenital heart disease are as yet unknown.

In this study we investigated plasma concentrations of BNP and ANP in asymptomatic or minimally symptomatic patients who had chronic right ventricular pressure overload. Plasma neurohormones were related to right ventricular ejection fraction, determined by magnetic resonance imaging (MRI). MRI is an accurate and reproducible method of determining various quantitative indices of right ventricular function in vivo. ${ }^{12} 13$

\section{Methods}

STUDY DESIGN AND PATIENT POPULATION

We studied 21 consecutive asymptomatic or minimally symptomatic outpatients (New York Heart Association functional class I and II) with chronic right ventricular pressure overload caused by congenital heart disease. Seven healthy volunteers matched for sex and age served as controls. Chronic right ventricular pressure overload was diagnosed by Doppler echocardiography (right ventricular systolic pressure $>35 \mathrm{~mm} \mathrm{Hg}$ ). All patients were studied off drug treatment. In each subject valvar 
Table 1 Clinical characteristics of the study population

\begin{tabular}{lll}
\hline & Patients & Volunteers \\
\hline Male/female & $12 / 9$ & $4 / 3$ \\
Age (years) & $26.6(8.6)$ & $26.9(3.9)$ \\
NYHA class I/II & $13 / 8$ & $7 / 0$ \\
ccTGA & 2 & \\
TGA (Mustard or Senning) & 9 & \\
Corrected tetralogy of Fallot & 10 & \\
Pulmonary artery stenosis & 2 & \\
RV systolic pressure (mm Hg) & 36 to 134 & $66.8(17.6)$ \\
RV EDV/BSA (ml/m $\left.{ }^{2}\right)$ & $65.3(23.3)$ & \\
\hline
\end{tabular}

Values are mean $(\mathrm{SD})$ or $\mathrm{n}$.

BSA, body surface area; ccTGA, congenitally corrected transposition of the great arteries; EDV, end diastolic volume; NYHA, New York Heart Association; RV, right ventricular; TGA, transposition of the great arteries.

regurgitation (>10 $\mathrm{ml}$ per cardiac cycle) was ruled out by MRI flow measurements. Patients with atrial arrhythmias were excluded from the study for two reasons: first; arrhythmias make MRI images unreliable for analysis because of the triggering problem; second, atrial arrhythmias are known to influence natriuretic peptide concentrations. ${ }^{14}$ Patients with chronic renal impairment (serum creatinine $>133 \mu \mathrm{mol} / \mathrm{l}$ ) or significant left ventricular abnormalities were also excluded. Informed consent was obtained from all patients and volunteers. The study was approved by the local ethics committee. Clinical characteristics of the study population are listed in table 1 .

IMAGING PROTOCOL

Study subjects were placed supine in a 1.5 Tesla MR imaging scanner (Vision, Siemens, Erlangen, Germany). MRI acquisition involved a standardised protocol. Imaging sessions were initiated with scout images to determine the position of the heart in the thoracic cavity. Based on these images an ECG triggered T1 weighted series of axial images was acquired. A gradient echo cine sequence was then performed in a plane bisecting the mitral valve orifice and passing through the apex, visualising the long axis view in order to localise the atrioventricular valve plane. An ECG triggered, ultrafast, breath held, gradient echo cine sequence (time of repetition $(T R)=R R$ interval, time of echo $(\mathrm{TE})=4.8 \mathrm{~ms}$, matrix size $=$ $256 \times 256$, field of view $(\mathrm{FOV})=350 \mathrm{~mm}$, flip angle $=20^{\circ}$ ) was then used to acquire images in the short axis plane in contiguous $10 \mathrm{~mm}$ slices encompassing the valve plane to the apex of the heart. Velocity maps were acquired with a flip angle of $30^{\circ}, \mathrm{TE}=5 \mathrm{~ms}$, slice thickness = $6 \mathrm{~mm}, \mathrm{FOV}=320 \mathrm{~mm}$, imaging matrix $=256$ $\times 256$, and velocity encoding $=250 \mathrm{~cm} / \mathrm{s}$.

IMAGE ANALYSIS

A Unix workstation was used for analysis of the magnetic resonance images. MASS (Medis, Leiden, Netherlands) image analysis software was used to display multislice, multiphase images individually and in a movie loop mode. End diastolic (maximum ventricular) and end systolic (minimum ventricular) volume frames were determined by manual outlining of a mid-ventricular slice in all phases. On end diastolic and end systolic time frames, endocardial borders of the right and left ventricles were outlined manually. Papillary muscles and the moderator band were not included in the ventricular volume measurements. The enclosed right ventricular and left ventricular cross sectional areas were measured by computer, multiplied by section thickness, and summed up according to Simpson's rule to provide left ventricular and right ventricular volumes.

Flow (Medis) image analysis software was used to calculate volumetric flow. Contours of the great artery arising from the right ventricle were drawn manually on the modulus images of all time frames. Flow $(\mathrm{ml} / \mathrm{s})$ per cardiac cycle was calculated using the velocity values of the corresponding velocity encoded images.

PLASMA NEUROHORMONES

Before the MRI examination, we obtained blood samples from the antecubital vein of all subjects after they had rested for at least 15 minutes. Blood was collected into chilled tubes containing EDTA and aprotinin $(1.9 \mathrm{mg}$ and $100 \mathrm{kIU} / \mathrm{ml}$ blood, respectively). The blood samples were promptly centrifuged $(1111 \mathrm{~g}$ for 10 minutes) and stored at $-70^{\circ} \mathrm{C}$ until final analysis. ANP and BNP concentrations were determined using immunoradiometric assay kits (Shionoria, Osaka, Japan).

\section{STATISTICAL ANALYSIS}

Differences between the groups were assessed by the unpaired Student's $t$ test. Probability values of $p<0.05$ were considered significant. All $\mathrm{p}$ values are two tailed. Linear regression analysis was performed to correlate neurohumoral factors and systolic right ventricular function. For descriptive purposes, quantitative variables with a normal distribution are presented as mean (SD).

\section{Results}

Right ventricular ejection fraction in the volunteers was higher than in the patients, at 69.0 $(8.2) \%$ v 58.0 (12.0)\%, respectively; $\mathrm{p}<0.006$ (fig 1A). Left ventricular ejection fraction was $72.3(7.8) \%$ in volunteers and $68.1(11.0) \%$ in patients (NS). There was a significant difference in the plasma concentrations of BNP and ANP between patients and volunteers-BNP, patients 5.3 (3.5) pmol/1 $v$ volunteers 2.3 (1.7) $\mathrm{pmol} / \mathrm{l}(\mathrm{p}<0.009)$; ANP, patients 7.3 $(4.5) \mathrm{pmol} / 1 \quad v$ volunteers 3.6 (1.4) pmol/1 $(\mathrm{p}<0.05)$ (fig 1B). In both patients and volunteers, mean plasma ANP concentrations were higher than mean plasma BNP concentrations. The right ventricular ejection fraction was inversely correlated with BNP $(r=0.65$; $\mathrm{p}<0.0002 ;$ fig 2A), and ANP $(r=0.61$; $\mathrm{p}<0.002$; fig $2 \mathrm{~B}$ ). No correlation was found between left ventricular ejection fraction and $\mathrm{BNP}(r=0.2 ; \mathrm{NS})$ or ANP $(r=0.52 ; \mathrm{NS})$. Similarly, no correlation was shown between the level of right ventricular systolic pressure and plasma BNP $(r=0.20 ; \mathrm{NS})$ or ANP $(r=0.07 ; \mathrm{NS})$. End diastolic right ventricular volume corrected for body surface area did not differ between patients and controls (65.3 (23.3) ข $66.8(17.6) \mathrm{ml} / \mathrm{m}^{2}$, respectively; NS). There were no differences in ventricular volumes between the different patient groups included in the study. 

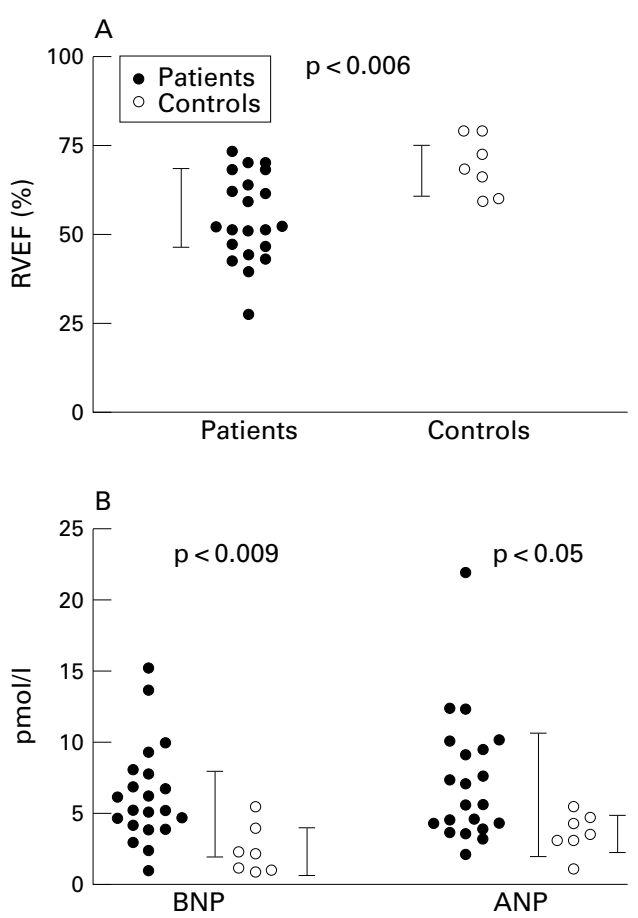

Figure 1 (A) Scatter graph showing right ventricular ejection fraction (RVEF) in patients and controls. (B) Scatter graph showing plasma concentrations of brain $(B N P)$ and atrial (ANP) natriuretic peptide in patients and controls. Values are means; error bars $=S D$.
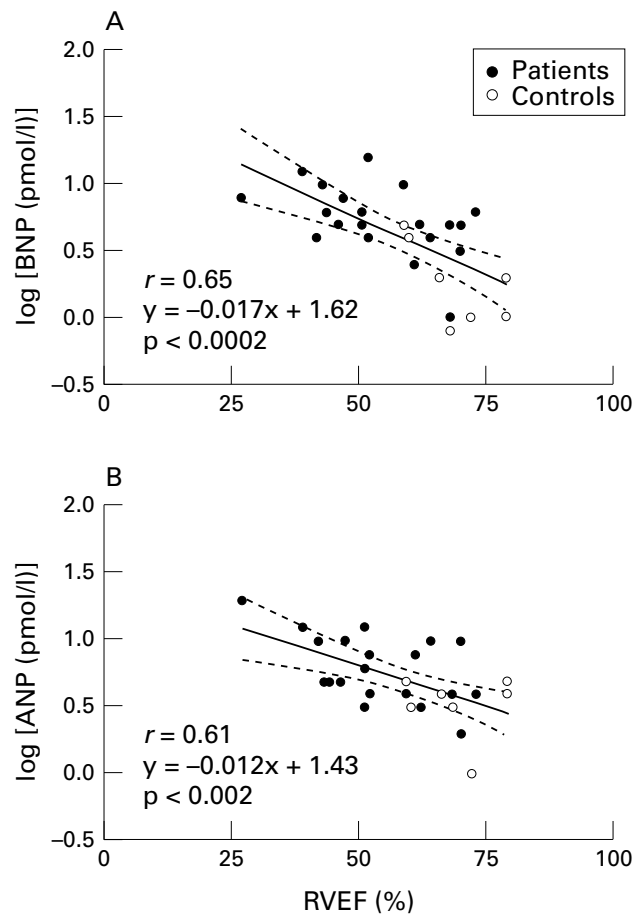

Figure 2 (A) Correlation between plasma brain natriuretic peptide (BNP) and right ventricular ejection fraction (RVEF). (B) Correlation between plasma atrial natriuretic peptide (ANP) and RVEF. Solid lines represent the linear regression and dotted lines are 95\% confidence intervals of the regression line.

\section{Discussion}

Our study showed that right ventricular ejection fraction was significantly lower in asymptomatic and minimally symptomatic patients with chronic right ventricular pressure overload than in healthy volunteers. In addition, concentrations of both BNP and ANP were significantly raised in the patients compared with the controls. We found an inverse correlation between right ventricular ejection fraction and the plasma neurohormones. There was no difference in left ventricular ejection fraction between patients and controls, and no correlation between left ventricular ejection fraction and the neurohormones.

To our knowledge, this is the first study to show a correlation between right ventricular systolic function and the neurohormones BNP and ANP in asymptomatic or minimally symptomatic patients with chronic right ventricular pressure overload from congenital heart disease.

Raised plasma concentrations of BNP and ANP have been reported before in patients with left ventricular systolic dysfunction. ${ }^{4} 1516$ Tsutamoto and colleagues stated that a high BNP may predict mortality and morbidity in asymptomatic patients with left ventricular dysfunction. ${ }^{17}$ Until now, the relation between neurohumoral factors and right ventricular function has received little attention. Only one previous study, by Nagaya and colleagues, ${ }^{10}$ showed increased BNP and ANP concentrations in symptomatic patients with right ventricular pressure and volume overload. In the current study, we observed increased plasma concentrations of natriuretic peptides in asymptomatic or minimally symptomatic patients with chronic right ventricular pressure overload.

Previous reports have indicated that in healthy subjects plasma ANP is higher than plasma $\mathrm{BNP}^{8}$ whereas in patients with manifest left or right ventricular failure BNP is increased substantially relative to ANP. ${ }^{6}{ }^{10}$ In our patients plasma BNP did not exceed plasma ANP, and concentrations of both neurohormones remained relatively low. This can be explained by the asymptomatic nature of early right ventricular dysfunction. On the basis of these data one could speculate that - as with left ventricular failure-plasma concentrations of BNP will start to exceed those of ANP as deterioration occurs from asymptomatic to symptomatic right ventricular failure.

No correlation was found between right ventricular systolic pressure and the plasma neurohormones, indicating that a rise in the plasma concentrations of these agents is a marker of right ventricular dysfunction rather than of increased right ventricular pressure in patients with congenital heart disease. This is consistent with the results of Nagaya and colleagues in patients with pulmonary hypertension. ${ }^{10}$

Despite the importance of detecting early right ventricular failure, the diagnosis of early asymptomatic right ventricular dysfunction remains difficult. ${ }^{2}$ Although non-invasive imaging techniques allow right ventricular function to be determined, these techniques are not suitable for large scale, cost effective application. Our data suggest that raised plasma BNP and ANP in asymptomatic or minimally symptomatic patients may be helpful as an early 
marker of right ventricular systolic dysfunction, and thus a potentially cost effective investigation in patients at risk of right ventricular failure. Monitoring changes in plasma BNP and ANP may provide quantitative follow up data in such patients.

Interpretation of plasma BNP concentrations must be done within an appropriate clinical context, because BNP may rise in various other pathological processes ${ }^{18}$ - for example, renal failure, ${ }^{19}$ significant left ventricular disease, ${ }^{4}$ and atrial arrhythmias. ${ }^{14}$

\section{CONCLUSIONS}

There is a significant inverse correlation between right ventricular ejection fraction and the plasma neurohormones BNP and ANP in asymptomatic or minimally symptomatic patients with chronic right ventricular pressure overload and congenital heart disease. Increased plasma BNP and ANP concentrations in these patients could be a useful early indicator of right ventricular systolic dysfunction, and monitoring changes in plasma BNP and ANP may provide quantitative follow up of right ventricular function. These results set the scene for larger studies of hormone guided follow up of asymptomatic patients with congenital heart disease who are at risk of right ventricular failure.

IIT is supported by the Netherlands Heart Foundation (NHS) (grant 9207) and Interuniversity Cardiology Institute of the Netherlands (ICIN-KNAW). DJvV is a clinical established investigator of the NHS

1 Graham TP. Ventricular performance in congenital heart disease. Circulation 1991;84:2259-74.

2 Oldershaw P, Bishop A. The difficulties of assessing right ventricular function. Br Heart $\mathcal{F}$ 1995;74:99-100.

3 Dell'Italia LJ, Santamore WP. Can indices of left ventricular function be applied to the right ventricle? Prog Cardiovasc Dis 1998;40:309-24.

4 McDonagh TA, Robb SD, Murdoch DR, et al. Biochemical detection of left-ventricular systolic dysfunction. Lancet 1998;351:9-13

5 Richards AM, Nicholls MG, Yandle TG, et al. Plasma $\mathrm{N}$-terminal pro-brain natriuretic peptide and adrenomedullin: new neurohormonal predictors of left ventricular function and prognosis after myocardial infarction. Circulation 1998;97:1921-9.

6 Mukoyama M, Nakao K, Hosoda K, et al. Brain natriuretic peptide as a novel cardiac hormone in humans. Evidence for an exquisite dual natriuretic peptide system, atrial natriuretic peptide and brain natriuretic peptide. $7 \mathrm{Clin}$ Invest 1991;87:1402-12.

7 Yasue $\mathrm{H}$, Yoshimura M, Sumida $\mathrm{H}$, et al. Localization and mechanism of secretion of B-type natriuretic peptide in comparison with those of A-type natriuretic peptide in normal subjects and patients with heart failure. Circulation 1994;90:195-203.

8 Van Veldhuisen DJ, Boomsma F, de Kam PJ, et al. Influence of age on neurohormonal activation and prognosis in patients

9 Van Veldhuisen DJ, Genth-Zotz S, Brouwer J, et al. Highversus low-dose ACE inhibition in chronic heart failure: a double-blind, placebo-controlled study of imidapril. $₹ \mathrm{Am}$ Coll Cardiol 1998;32:1811-18.

10 Nagaya N, Nishikimi T, Okano Y, et al. Plasma brain natriuretic peptide levels increase in proportion to the extent of right ventricular dysfunction in pulmonary hypertension. $\mathcal{F}$ Am Coll Cardiol 1998;31:202-8.

11 Matsuo K, Nishikimi T, Yutani C, et al. Diagnostic value of plasma levels of brain natriuretic peptide in arrhythmogenic right ventricular dysplasia. Circulation 1998;98: 2433-40.

12 Pattynama PM, De Roos A, Van der Wall EE, et al. Evaluation of cardiac function with magnetic resonance imaging. Am Heart f 1994;128:595-607.

13 Rebergen SA, van der Wall EE, Doornbos J, et al. Magnetic resonance measurement of velocity and flow: technique, validation, and cardiovascular applications. Am Heart $\mathcal{f}$ 1993;126:1439-56.

14 Berglund H, Boukter S, Theodorsson E, et al. Raised plasma concentrations of atrial natriuretic peptide are independent of left atrial dimensions in patients with chronic atrial fibrillation. Br Heart f 1990;64:9-13.

15 Lerman A, Gibbons RJ, Rodeheffer RJ, et al. Circulating $\mathrm{N}$-terminal atrial natriuretic peptide as a marker for symptomless left-ventricular dysfunction. Lancet 1993;341: tomless

16 Talwar S, Squire IB, Davies JE, et al. Plasma N-terminal pro-brain natriuretic peptide and the ECG in the assessment of left-ventricular systolic dysfunction in a high risk population. Eur Heart f 1999;20:1736-44.

17 Tsutamoto T, Wada A, Maeda K, et al. Plasma brain natriuretic peptide level as a biochemical marker of morbidity and mortality in patients with asymptomatic or minimally ymptomatic left ventricular dysfunction-comparison with plasma angiotensin II and endothelin-1. Eur Heart 7 with plasma angiotens

18 Cowie MR, Struthers AD, Wood DA, et al. Value of natriuretic peptides in assessment of patients with possible new heart failure in primary care. Lancet 1997;350:1349-53.

19 Lang CC, Choy AM, Henderson IS, et al. Effect of haemodialysis on plasma levels of brain natriuretic peptide in patients with chronic renal failure. Clin Sci 1992;82:127- 